\title{
ZONE ESTIMATES IN THE ELASTIC-PLASTIC TORSION PROBLEM*
}

\author{
By WAN-LEE YIN (National Tsing Hua University, Hsinchu, Taiwan)
}

Summary. Using continuity and jump conditions across the elastic-plastic interface, we show that the curvature of the shearing stress lines in the plastic zone of a simply connected bar under torsion is bounded above by a number proportionate to the twisting angle. For sufficiently large torsion the above geometrical condition defines a region in the cross-section which is a priori elastic. This lower bound for the elastic zone in the sense of set inclusion is supplemented by an upper bound for the zone area.

1. Introduction. We consider torsion of a cylindrical bar of an elastic, perfectly plastic material having a simply connected cross-section $\Omega$. It is well known that the bar becomes fully plastic only in the limit when $\alpha$, the angle of twist per unit length of the bar. becomes infinitely large. This fully plastic state is distinguished by the occurrence of lines of discontinuity of stress-the ridge lines in the sandhill analogy. For any finite value of $\alpha$, there is always an elastic zone $\Omega_{e}(\alpha)$ in $\Omega$ and this elastic zone includes the ridge in its interior. We may therefore regard the ridge as the ultimate barrier to the growth of the plastic zone. This barrier depends only on the cross-section $\Omega$ and can be obtained geometrically.

To obtain a better result, we show that if the boundary of $\Omega$ contains a smooth convex arc, then for each sufficiently large value of $\alpha$, it is possible to construct geometrically a subregion $\Omega_{\alpha}$ of the elastic zone $\Omega_{e}(\alpha)$ with positive area. This "a priori elastic region" is defined by the following geometrical condition: in $\Omega_{\alpha}$ the curves parallel to the boundary of $\Omega$ (i.e. the shearing stress lines for the fully plastic solution) are convex and have curvatures greater than $2 G \alpha / k$, where $G$ is the elastic shear modulus and $k$ is the yield stress in pure shear. Thus, although we cannot know the elastic-plastic interface for any given value of $\alpha$ without actually solving the torsion problem, we know that the elastic zone $\Omega_{e}(\alpha)$ includes both $\Omega_{\alpha}$ and the ridge. The defining condition of $\Omega_{\alpha}$ implies that a sufficiently large ratio of curvature to $\alpha$ on a convex part of the boundary prevents yielding in the vicinity of the part, irrespective of the shape of the cross-section away from that vicinity.

The area of the a priori elastic region is a lower bound for the area of the elastic zone. An upper bound for the latter can be obtained by means of the divergence theorem, as shown in Sec. 4.

2. Review of the elastic-plastic torsion problem. In its mathematical formulation, the elastic-plastic torsion problem requires the determination, for each positive number $\alpha$, of a continuously differentiable function $\phi(x, y)$ vanishing along the boundary of the domain $\Omega$ and satisfying in $\Omega$ either one of the following two conditions [1]:

$$
|\nabla \phi|=k \text {, }
$$

* Received March 17, 1977. 
or

$$
|\nabla \phi|<k \text { and } \nabla^{2} \phi=-2 G \alpha .
$$

We say that (1) is valid in the plastic zone $\Omega_{p}(\alpha)$ and (2) is valid in the elastic zone $\Omega_{e}(\alpha)$.

If $G \alpha / k$ is sufficiently small, a solution of $(2)$ exists in the whole domain $\Omega$ and there is no plastic zone. For larger values of $\alpha$, our definition of the problem requires the continuity of $\nabla \phi$ across the elastic-plastic interface $\Gamma$.

As stated in the introduction, we shall assume the domain $\Omega$ simply connected, where we let

$$
\nabla \phi=-\tau \mathbf{e}, \quad|\mathbf{e}|=1 \text {; }
$$

then in the plastic zone the magnitude of shearing stress $\tau$ has the constant value $k$ and the trajectories of the unit vector field e are straight lines:

$$
(\mathbf{e} \cdot \nabla) \mathbf{e}=\left(2 k^{2}\right)^{-1} \nabla(\nabla \phi \cdot \nabla \phi)=\left(2 k^{2}\right)^{-1} \nabla\left(k^{2}\right)=0 .
$$

The orthogonal trajectories of the rectilinear e-lines are parallel curves having constant $\phi$ values. These curves are called shearing stress lines since their tangents agree with the direction of the shearing stress vector. In the elastic zone the e-lines are not rectilinear and the shearing stress lines are not parallel curves.

In order to have a single-valued displacement function, it is necessary that in the plastic zone each rectilinear e-line does not intersect the interface $\mathrm{I}$ more than once [2]. Consequently, starting from some point on $\Gamma$, an e-line remains in the plastic zone until it intersects the boundary $\phi=0$ orthogonally. Thus each component of the plastic zone is adjacent to the boundary of $\Omega$, and the outward normal $\mathbf{n}$ from the elastic zone $\Omega_{e}$ satisfies

$$
\mathrm{n} \cdot \mathrm{e} \geq 0 \text { on } \Gamma \text {. }
$$

3. A priori elastic zone. From (2) and (3) we obtain

$$
\begin{aligned}
\nabla^{2}\left(\tau^{2} / 2\right)= & \phi_{x x}{ }^{2}+\phi_{y y}{ }^{2}+2 \phi_{x y}{ }^{2}=\left(\phi_{x x}-\phi_{y y}\right)^{2} / 2 \\
& +\left(\phi_{x x}+\phi_{y y}\right)^{2} / 2+2 \phi_{x y}{ }^{2} \geq 2\left(G^{2} \alpha^{2}+\phi_{x y}{ }^{2}\right)>0 \quad \text { in } \Omega_{e},
\end{aligned}
$$

where the subscripts $x$ and $y$ denote partial differentiation with respect to the coordinates. In $\bar{\Omega}_{e}$ the magnitude of shearing stress $\tau$ reaches its maximum value $k$ along the interface I. Applying a maximum principle [3] to (5), we obtain

$$
\lim _{x \rightarrow \Gamma^{e}} n \cdot \nabla\left(\tau^{2} / 2\right)>0 \text { on } \Gamma,
$$

where $\mathbf{x} \rightarrow \Gamma^{e}$ indicates taking the limit from the elastic side (the corresponding limit from the plastic side is obviously zero). Consequently,

$$
\lim _{\mathbf{x} \rightarrow \Gamma^{e}} \nabla \tau=\lim _{\mathbf{x} \rightarrow \Gamma^{e}}|\nabla \tau| \mathrm{n} \quad \text { on } \Gamma .
$$

Hence (4) implies

$$
\lim _{\mathbf{x} \rightarrow \Gamma^{e}} \mathbf{e} \cdot \nabla \tau=\lim _{\mathbf{x} \rightarrow \Gamma^{e}}|\nabla \tau| \mathbf{n} \cdot \mathbf{e} \geq 0 \quad \text { on } \Gamma .
$$

Using (3), we write (2) in the form

$$
\tau \nabla \cdot \mathbf{e}+\mathbf{e} \cdot \nabla \boldsymbol{\tau}=2 G \alpha \quad \text { in } \Omega_{e} .
$$


In the limit $\mathbf{x} \rightarrow \Gamma^{e}$, we have

$$
k \lim _{\mathbf{x} \rightarrow \Gamma^{e}} \nabla \cdot \mathbf{e}+\lim _{\mathbf{x} \rightarrow \Gamma^{e}} \mathbf{e} \cdot \nabla \tau=2 G \alpha .
$$

Hence (7) implies that

$$
\lim _{\mathbf{x} \rightarrow \Gamma^{e}} \nabla \cdot \mathbf{e} \leq 2 G \alpha / k
$$

In $\Omega$, we define $\theta$ and $\mathrm{f}$ by

$$
\mathbf{e}=\cos \theta \mathbf{i}+\sin \theta \mathbf{j}, \quad \mathbf{f}=-\sin \theta \mathbf{i}+\cos \theta \mathbf{j},
$$

where $\mathrm{i}$ and $\mathrm{j}$ are the unit vectors in the $x$ and $y$ directions.

Then,

$$
\begin{aligned}
\nabla \cdot \mathbf{e} & =-\sin \theta \theta_{x}+\cos \theta \theta_{y}=\mathbf{f} \cdot \nabla \theta \\
-\nabla \cdot \mathbf{f} & =\cos \theta \theta_{x}+\sin \theta \theta_{y}=\mathbf{e} \cdot \nabla \theta
\end{aligned}
$$

are the curvatures of shearing stress lines and their orthogonal trajectories. These curvatures are in general discontinuous across the interface $\Gamma$, since the rectilinear e-lines become curvilinear as they pass from $\Omega_{p}$ to $\Omega_{e}$. We now derive a relation between the jumps of these curvatures across $\Gamma$.

On the interface we further define $\beta$ and $t$ by

$$
\mathbf{n}=\cos \beta \mathbf{e}-\sin \beta \mathbf{f}, \quad \mathbf{t}=\sin \beta \mathbf{e}+\cos \beta \mathbf{f},
$$

Then $\mathrm{t}$ is tangential to the interface and (4) implies that

$$
\cos \beta \geq 0 \text {. }
$$

Since $\theta$ is continuous across $\Gamma$, its tangential derivative $t \cdot \nabla \theta$ is also continuous across $\Gamma$, where

$$
\begin{aligned}
\mathbf{t} \cdot \nabla \theta & =\sin \beta \mathbf{e} \cdot \nabla \theta+\cos \beta \mathbf{f} \cdot \nabla \theta \\
& =-\sin \beta \nabla \cdot \mathbf{f}+\cos \beta \nabla \cdot \mathbf{e} .
\end{aligned}
$$

Thus $\sin \beta(\nabla \cdot \mathbf{f})$ and $\cos \beta(\nabla \cdot \mathbf{e})$ suffer the same amount of jump across the interface.

The shearing stress lines $\phi=$ constant have maximum density in $\Omega_{p}(|\nabla \phi|=k)$. These curves diverge as they enter the elastic zone. Since $f$ is their unit tangent vector, we have on the plastic side $\nabla \cdot \mathrm{f}=0$ and on the elastic side of $\Gamma$

$$
\nabla \cdot \mathrm{f}\left\{\begin{array}{l}
\geq 0 \text { if } \mathrm{f} \cdot \mathrm{n}=-\sin \beta \leq 0, \text { i.e., if } \mathrm{f} \text { is toward } \Omega_{e}, \\
\leq 0 \text { if } \mathrm{f} \cdot \mathrm{n} \geq 0 \text {, i.e., if } \mathrm{f} \text { is toward } \Omega_{p},
\end{array}\right.
$$

or equivalently

$$
\sin \beta \nabla \cdot \mathrm{f} \geq 0 .
$$

Thus from $\Omega_{p}$ to $\Omega_{e}$ the jump in $\sin \beta(\nabla \cdot \mathbf{f})$ is non-negative. By continuity of (12) and according to (11) the jump in $\nabla \cdot \mathbf{e}$ is also positive, i.e.

$$
\lim _{\mathbf{x} \rightarrow \Gamma^{p}} \nabla \cdot \mathbf{e} \leq \lim _{\mathbf{x} \rightarrow \Gamma^{e}} \nabla \cdot \mathbf{e} \text { on } \Gamma,
$$

where $\mathbf{x} \rightarrow \Gamma^{p}$ indicates taking limit from the plastic side. From (10) and (13) we finally obtain 


$$
\lim _{\mathbf{x} \rightarrow \Gamma^{p}} \nabla \cdot \mathbf{e} \leq 2 G \alpha / k \text { on } \Gamma .
$$

As mentioned above, $\nabla \cdot \mathbf{e}$ is the curvature of lines of shearing stress and in the plastic zone these curves are parallel to the boundary of the cross-section. If a point is in the plastic zone $\Omega_{p}$, this point must be on the elastic-plastic interface for some smaller value of $\alpha$, say, $\alpha_{1}$. Then we have at that point

$$
\nabla \cdot \mathbf{e} \leq 2 G \alpha_{1} / k<2 G \alpha / k .
$$

This implies that if through a point of $\Omega$ the curve parallel to the boundary is convex and has curvature greater than $2 G \alpha / k$, then the point cannot be in $\bar{\Omega}_{p}$, and hence must be in $\Omega_{e}$. The set of all such points defines the a priori elastic region at the twisting angle $\alpha$, as stated in the introduction.

If the boundary of $\Omega$ does not contain a smooth convex arc, i.e. if the boundary consists of line segments and concave arcs, then the curves parallel to the boundary also have non-negative curvature and there is no a priori elastic region for any $\alpha$.

4. Upper bound for the area of the elastic zone. Since $\phi$ and its first derivatives are continuous in $\Omega$ and the second derivatives are piecewise continuous, we may apply the divergence theorem to the region $\Omega$ :

$$
-\iint_{\Omega} \nabla^{2} \phi d x d y=-\oint_{\partial \Omega} \mathbf{e} \cdot \nabla \phi d s=\oint_{\partial \Omega} \tau d s
$$

where $s$ denotes the arc length along the boundary $\partial \Omega$ and the last equality follows from (3). The area integral can be decomposed into two parts: the one over $\Omega_{e}$

$$
-\iint_{\Omega_{e}} \nabla^{2} \phi d x d y=2 G \alpha A_{e},
$$

where $A_{e}$ is the area of $\Omega_{e}$; the other over $\Omega_{p}$

$$
-\iint_{\Omega_{p}} \nabla^{2} \phi d x d y=k \iint_{\Omega_{p}} \nabla \cdot \mathbf{e} d x d y
$$

In $\Omega_{p}$ the curves parallel to the boundary have curvature $\nabla \cdot \mathbf{e}$. Hence $\nabla \cdot \mathbf{e}$ has the same sign along each rectilinear e-line, i.e. non-negative if the line intersects the boundary at a convex or flat point and negative if otherwise. If the boundary adjacent to a component of $\Omega_{p}$ consists of alternative concave and non-concave parts, then that component of $\Omega_{p}$ is divided by several rectilinear e-lines into regions where $\nabla \cdot \mathbf{e}$ is alternatively negative and non-negative. To each part of $\Omega_{p}$ with negative $\nabla \cdot \mathbf{e}$, as shown in Fig. 1, we extend the

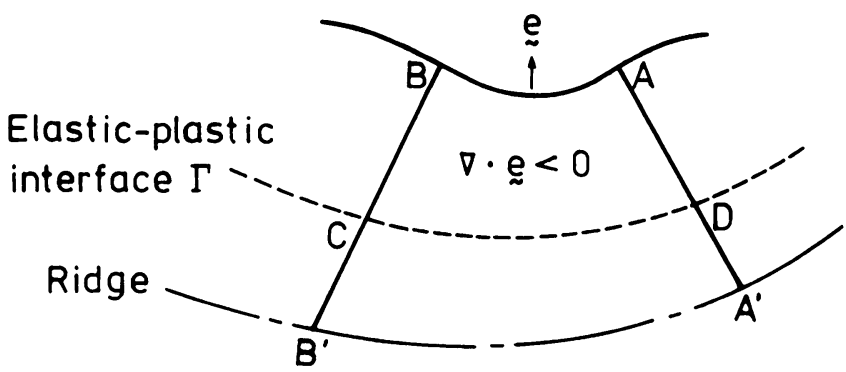

FIG. 1. 
domain of integration of $\iint \nabla \cdot \mathbf{e} d x d y$ to the ridge line, i.e., from $A B C D$ to $A B B^{\prime} A^{\prime}$, and then apply the divergence theorem. We obtain

$$
\iint_{A B C D} \nabla \cdot \mathbf{e} d x d y>\iint_{A B B^{\prime} A^{\prime}} \nabla \cdot \mathbf{e} d x d y=\int_{A B} d s-\int_{A^{\prime} B^{\prime}} \mathrm{n}^{\prime} \cdot \mathbf{e} d s,
$$

where $\mathrm{n}^{\prime}$ is the unit normal vector along the ridge satisfying $\mathrm{n}^{\prime} \cdot \mathbf{e}>0$.

For the integral on the right-hand side of (17) we discard the contribution from the area with positive integrand. We then substitute (16) and (17) into (15) and use (18) and $\tau$ $\leq k$, obtaining

$$
(2 G \alpha / k) A_{e} \leq \oint_{\partial \Omega} d s-\sum \int_{A^{\prime} B^{\prime}} \mathrm{n}^{\prime} \cdot \mathbf{e} d s,
$$

where the summation is over all concave boundary arcs such as $A B$ and where $A^{\prime} B^{\prime}$ is the segment of ridge line facing $A B$. A somewhat simpler but cruder upper bound for $(2 G \alpha / k) A_{e}$ is the sum of the total arc length of non-concave parts of $\partial \Omega$ and the total arc length of ridge lines facing concave parts of $\partial \Omega$. If the boundary of the cross-section has no concave part, then (19) reduces to

$$
A_{e} \leq(k / 2 G \alpha) \oint_{\partial \Omega l} d s .
$$

To be useful at all, an upper bound for $A_{e}$ must be smaller than the area of the whole cross-section. This is the case whenever $\alpha$ is sufficiently large.

\section{REFERENCES}

[1] R. Hill, The mathematical theory of plasticity, Oxford, 1950, pp. 84-89

[2] W. Prager and P. G. Hodge, Jr., Theory of perfectly plastic solids, Wiley, New York, 1951, p. 68

[3] L. Bers, F. John and M. Schechter, Partial differential equations, Interscience, New York, 1964, p. 151 